\title{
Dent Damage Identification in Stiffened Cylindrical Structures Using Inverse Finite Element Method
}

\author{
Mingyang $\mathrm{Li}^{1}$, Adnan $\mathrm{Kefal}^{2,3}$, Burak Can $\mathrm{Cerik}^{4}$, and Erkan Oterkus ${ }^{1, *}$ \\ ${ }^{1}$ Department of Naval Architecture, Ocean and Marine Engineering, University of Strathclyde, Glasgow, UK \\ ${ }^{2}$ Faculty of Naval Architecture and Ocean Engineering, Istanbul Technical University, Istanbul, Turkey \\ 3Integrated Manufacturing Technologies Research and Application Center, Sabanci University, Tuzla, 34956, \\ Istanbul, Turkey \\ ${ }^{4}$ Department of Naval Architecture and Ocean Engineering, Inha University, Incheon, Korea
}

\begin{abstract}
The offshore industry has been using stiffened thin-walled steel cylindrical structures for decades, particularly as the columns of floating offshore installations. The floating offshore installations may be subjected to severe marine environmental conditions. Accidents such as collisions may also occur. Structural Health Monitoring (SHM) is a viable tool to maintain safe operation of offshore installations. Inverse Finite Element Method (iFEM) is one of the most powerful methods for SHM process. Hence, this study focuses on the application of iFEM methodology to thin-walled cylindrical structures representing the columns of floating offshore installations. iFEM methodology is verified by comparing its displacement results against reference finite element method (FEM) solution. After this verification, four different damage cases with different size, location and number of damages are considered. By using a newly introduced damage parameter and von Mises strain distribution iFEM accurately identified the correct damage locations and sizes. Therefore, it is concluded that iFEM can be used for structural damage prediction in offshore structures with high accuracy even if the number of the strain sensors is limited.
\end{abstract}

Keywords: Structural health monitoring; Inverse finite element method; Stiffened cylinder; Floating offshore installations

\section{Introduction}

In order to keep the engineering structures safe and reliable, detecting an unusual structural condition, such as fatigue cracking, dents induced by mass impact, age-related degradation, is crucial in all engineering fields. The use of structural health monitoring (SHM) systems to perform continuous monitoring of structures is a viable option from this aspect. SHM requires installation of sensing systems into the structure to collect the required real-time data. After processing the data, the health condition of the structure can be obtained [1]. SHM is widely applied to the estimation of the life of the structures and can allow determination of optimum periods for inspection and repair [2]. Besides, SHM is essential for reducing the risks and protecting the structures from failures, which eventually keeps human and environment safe.

Most of the current approaches in SHM cannot be used directly for predicting 3-Dimensional displacement and stress fields because complex boundary conditions and topology of the structures 
cannot be fully considered. To overcome this problem, real-time shape sensing can play an important role in the development of SHM systems especially for structures with complex loading conditions. The main benefit of shape sensing is that it allows to use the measured strain data to estimate the variation of the displacement field in the structure [3]. This data can then be used to determine strains and stresses for SHM systems. Currently, traditional strain rosettes and embedded fiber-optic sensor networks including Fiber Bragg Grating (FBG) sensors are commonly used to collect the required strain data. Conventional strain rosettes have benefits of convenience and inexpensive price. On the other hand, FBG sensors have been broadly used in many fields including the wings of aircrafts, complex frame structures and composite materials [4-7].

According to Gherlone et al. [8], the shape sensing should, in the first place, be able to take into account the complex geometry and boundary conditions of the structures. Moreover, it should provide reliable and stable displacement data even in the case of unavoidable errors occurring during the measurement of the strain values. Finally, shape sensing should be fast enough to ensure the necessary real-time monitoring purposes. Among various shape sensing methods, the typical strategies are Ko's Displacement Theory, Modal Method (MM) and inverse Finite Element Method (iFEM) [3]. Ko's Displacement Theory was introduced by Ko et al. [11] based on twice integration of measured axial strains from sensors which are located on a different sensing line rather than the traditional axis [11]. Ko's Displacement theory is based on classical beam equations and it is very suitable for beam type structures. The accuracy of this approach has been proven by FEM comparisons [11]. Its usage has been extended to wing-boxes and plates using multi-plex sensing lines [4,6].

On the other hand, depending on the mode shapes of the structure, MM uses the measured strains to calculate the displacements according to the relationships between strains and displacements [12]. The main advantage of $\mathrm{MM}$ is that it can make predictions without knowing its material properties and it can be used to analyse beam and plate type structures. However, MM is quite sensitive to frequency and amount of strain sensors [16,17]. As an alternative approach, iFEM is an advanced and powerful concept developed by Tessler and Spangler $[18,19]$. iFEM discretizes the whole structure into suitable inverse finite elements (beam, plate, shell and solid elements) and the only input data is the strain of each element. iFEM takes into account all strain components including membrane, bending and transverse shear depending on the element type and structure. It utilises least square methodology to minimise differences between measured and numerical strains. This process leads to a system of equations in matrix form. By solving the matrix system, the displacements at each node of iFEM elements can be determined at any real time. After the calculation of the displacements at each node, the strains of the whole structure can be estimated which will then contribute to the evaluation of the stress distribution of the structure. In short, iFEM only uses strain data collected from strain sensors to generate full-field displacements, strains and then stresses.

Gherlone et al. [3] has recently compared the three main shape shaping methods using a cantilevered wing-shaped aluminium plate under static conditions. The accuracy of these three methods is investigated by comparing against experimental results [3]. After comparison, it was concluded that iFEM is more flexible and accurate than the other two techniques [3]. Besides, iFEM doesn't require information about loading conditions.

There has been a steady progress on iFEM studies in the literature. Amongst these Tessler and Spangler [20] developed a three-node inverse shell element, iMIN3, which has linear in-plane displacements and bending rotations, and quadratic transverse-displacement (deflection) along in- 
plane coordinates. To interpolate these displacement fields, Tessler and Spangler [20] utilized the linear area-parametric coordinates of a triangle and its associated anisoparametric shape functions developed based on constant shear-edge constraint condition [21]. Quach et al. [22] and Vazquez et al. [23] proved the reliability and capability of the iMIN3 element by performing an experimental study. Additionally, Tessler et al. [24] improved the iMIN3 element to make it suitable for large deformations occurring in the plate and shell structures. For beam type structures, Cerrachio et al. [25] and Gherlone et al. $[8,26,27]$ developed an iFEM beam element based on Timoshenko beam formulation. The accuracy of their element has been tested successfully through numerical and experimental studies. For complex materials, Cerracchio et al. [28,29] developed a robust plate element for composite and sandwich structures which then became the foundation for Kefal et al. [1] to extend it to a new shell element called i3-RZT for composite materials. Moreover, Kefal et al. [30,31] developed a four-node quadrilateral iFEM element, iQS4. The iQS4 element is based on 1st order transverse-shear deformation theory having six degrees of freedom (DOF) including drilling DOF. The iQS4 element has a shape sensing capability for both thin or thick shell structures. The accuracy and practicability of iQS4 element has been demonstrated by Kefal et al. [30,31].

Offshore structures often suffer from extreme loading conditions including wave loads and wind loads. In addition, they may also experience abnormal and accidental loadings such as impact due to ice, attending vessels (collision), slamming, and dropped objects. Moreover, steel, which is the most common material used for offshore installations, can suffer from corrosion caused by sea water. Consequently, it is essential to utilize SHM systems to monitor the health of offshore structures. Since the International Maritime Organization released suggestions to spread SHM for the safety of ship structures, several applications have been performed using different kinds of SHM systems for different ship types [32-36]. As an alternative approach, Kefal and Oterkus [37, 38] applied iFEM/iQS4 for complex marine structures such as Panamax containership and chemical tanker. The results show that iFEM can be simply and efficiently used for the SHM of ship structures with robust, fast and reliable results. 


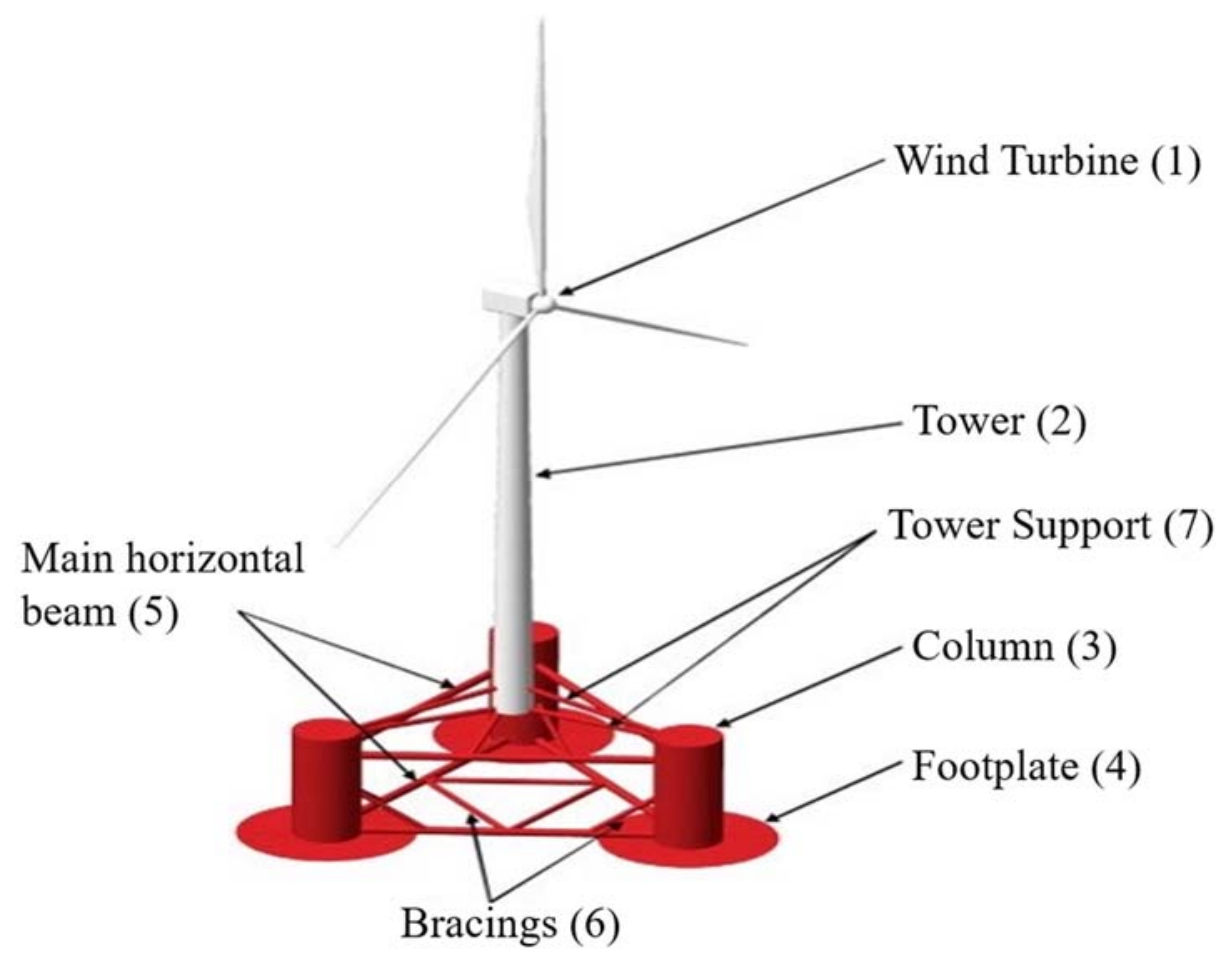

Figure 1. Tri-floater offshore wind turbine with stiffened cylinders as main columns [43]

Stiffened thin-walled cylindrical structures are one of the most common structural components of floating offshore installations [39-43]. Fig. 1 shows a typical application of a floating offshore wind turbine where the columns are orthogonal stiffened cylinders. Considering these structures being commonly subjected to severe environmental conditions, it is necessary to use SHM system to protect the structure from failure. Therefore, the current study presents the application of iFEM approach to identify dent damage in a thin-walled stiffened steel cylinderical structure. The present paper is organized as follows: in section 2 the iFEM methodology and iQS4 element are briefly explained. In the next section, the properties of the cylindrical structures considered in this study are provided. Moreover, the numerical results for undamaged and damaged cylindrical structure cases are demonstrated. The damage prediction in the stiffened cylindrical structures using a newly introduced damage parameter and von Mises strain distributions is also presented.

\section{The iFEM Methodology}

\subsection{Basics of iFEM Methodology}

In the iFEM methodology, the geometry of the structure to be monitored is discretised by using suitable inverse elements as shown in Fig. 2(a). To perform an experimental application of iFEM methodology for a given structure, triaxial strain measurements should be collected from discrete locations of the top/bottom faces of the structural components. For this purpose, three different conventional strain gauges and/or embedded FBG sensors can be stacked together to form a strain rosette, which can mounted on a discrete position to obtain onboard triaxial strain measurements in real time. Once the data raw data is collected from each strain gauge, it has to be sampled according to 
the desired frequency interval through an averaging/filtering process. After that, for a particular position, triaxial strain measurements should be transformed to normal strain components and in-plane shear strain of the local iFEM element. This transformation can be readily performed by utilizing direction angles between local axes of the element and the strain-gauge axis.

In the current study, we generated the discrete (experimental) strain data numerically through performing a high-fidelity finite element analysis on the structures concerned. For this purpose, a relatively dense finite element model is created first. Secondly, the material, loading, and constraint conditions are assigned to the dense FEM model. Third, the nodal displacement and rotation solutions are obtained after the analysis. In the post-processing stage of the FEM analysis, the direct input of iFEM analysis, i.e., discrete normal/shear strain measurements over the local axes of the iQS4 element, are produced by multiplying the displacement and rotation solutions of the high-fidelity FEM model together with the derivatives of the associated FEM element shape functions. As a result, this numerical strain data represents experimental strain readings obtained from surface patched strain gauges or FBG sensors along the local axes of the iQS4 element. Processing the in-situ strain data collected from discrete locations of the structure, full field displacements, strains and stresses can be obtained in real time as depicted in Fig. 2(b).
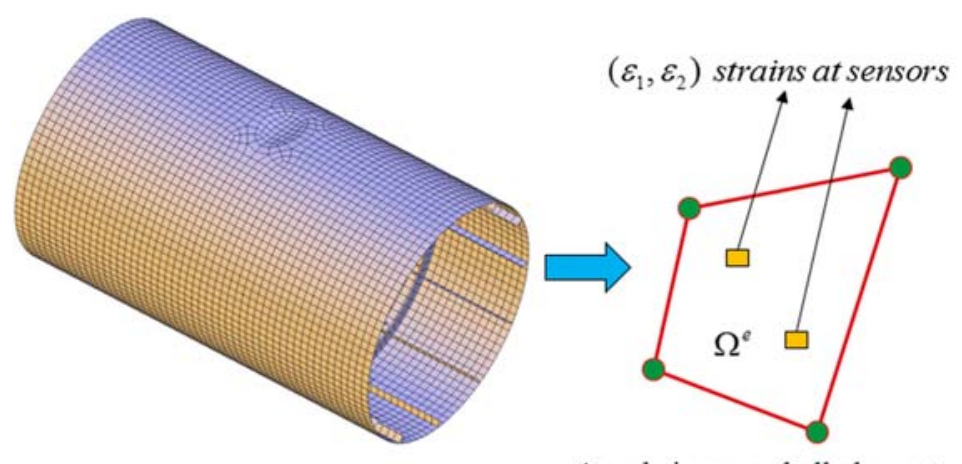

(a) 


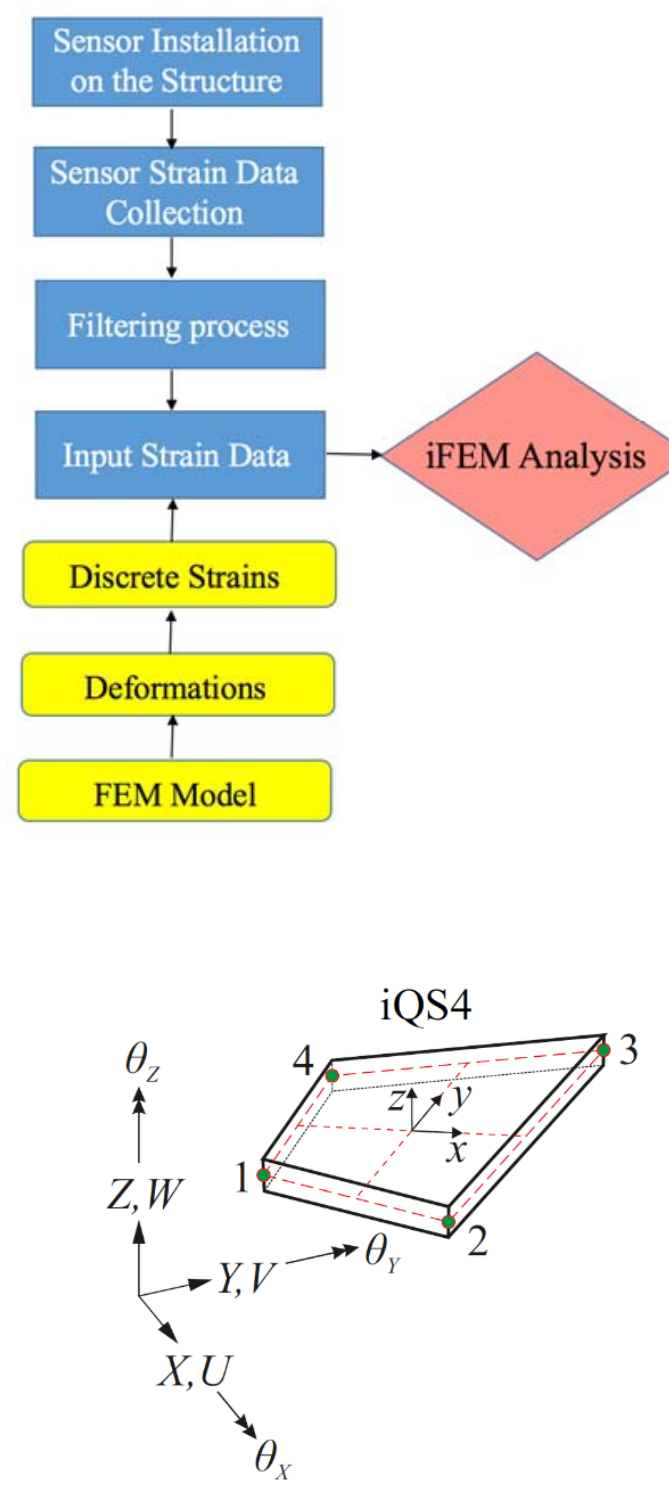

(c)

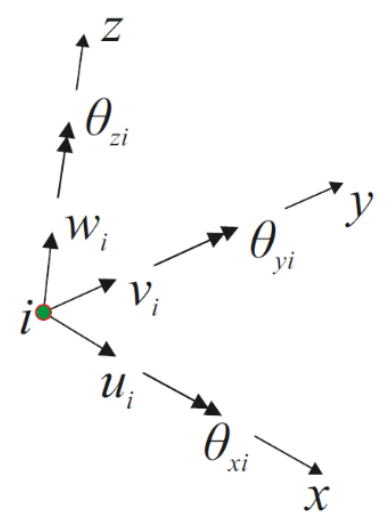

(d)

Figure 2. (a) Discretisation of the structure with inverse finite elements, (b) overview of iFEM methodology, (c) iQS4 element and (d) nodal DOF [1]

\subsection{Formulation of iQS4 element and analytical strains}

The iQS4 element developed by Kefal et al. [31] is a four-node quadrilateral element with a thickness of $2 h$ as shown in Fig. 2(c). For this element, the top and bottom surface of the shell correspond to the thickness coordinates of $z=+h$ and $z=-h$, respectively, such that $z \in[-h,+h]$. As depicted in Fig. 2(d), the iQS4 element has six degrees-of-freedom (DOF) at each node. Utilizing these DOF, the in-plane translations $u$ and $v$ along positive $x$ and $y$ axes and also transverse deflection $w$ along positive $z$ axis are interpolated using quadratic anisoparametric shape functions, whereas the bending rotations $\theta_{i}(i=x, y)$ are approximated based on bilinear isoparametric shape functions. For the brevity of the paper, these shape functions are not given here. However, the exact expressions of these shape functions and related interpolation of the kinematic variables can be found 
in [31]. According to the linear strain-displacement relationship given in [31], three different matrices that contain the derivatives of shape functions can be constructed for each individual strain measure, namely, $\boldsymbol{B}^{m}$ matrix for the membrane strains, $\boldsymbol{B}^{b}$ matrix for bending curvatures, and $\boldsymbol{B}^{s}$ matrix for transverse shear strains. The explicit form of these matrices are also provided in [31] and not included herein for conciseness. These matrices relate the membrane strains, $\boldsymbol{e}$, bending curvatures, $\boldsymbol{\kappa}$, and transverse shear strains, $\boldsymbol{\gamma}$, to the nodal displacements and rotations which are given as [31]:

$\boldsymbol{e}=\left[\begin{array}{lll}e_{1} & e_{2} & e_{3}\end{array}\right]^{T}=\boldsymbol{B}^{m} \boldsymbol{u}_{e}$

$\boldsymbol{\kappa}=\left[\begin{array}{lll}\kappa_{1} & \kappa_{2} & \kappa_{3}\end{array}\right]^{T}=\boldsymbol{B}^{b} \boldsymbol{u}_{e}$

$\boldsymbol{\gamma}=\left[\begin{array}{ll}\gamma_{1} & \gamma_{2}\end{array}\right]^{T}=\boldsymbol{B}^{s} \boldsymbol{u}_{e}$

where the vector $\boldsymbol{u}_{e}=\left[\begin{array}{lll}\boldsymbol{u}_{1}^{e} & \cdots & \boldsymbol{u}_{4}^{e}\end{array}\right]^{T}$ represents all nodal DOF of the iQS4 element with $\boldsymbol{u}_{i}^{e}=\left[\begin{array}{llllll}u_{i} & v_{i} & w_{i} & \theta_{x i} & \theta_{y i} & \theta_{z i}\end{array}\right]^{T}(i=1, \ldots, 4)$ denoting the six DOF at each node (Fig. 2d). Utilizing the strain measures given in Eq. (1), the in-plane and transverse-shear strain components at any point given in the plate geometry can be calculated as:

$\left\{\begin{array}{l}\varepsilon_{x x} \\ \varepsilon_{y y} \\ \gamma_{x y}\end{array}\right\}=\left\{\begin{array}{l}e_{1} \\ e_{2} \\ e_{3}\end{array}\right\}+z\left\{\begin{array}{l}\kappa_{1} \\ \kappa_{2} \\ \kappa_{3}\end{array}\right\}$

and

$\left\{\begin{array}{l}\gamma_{x z} \\ \gamma_{y z}\end{array}\right\}=\left\{\begin{array}{l}\gamma_{1} \\ \gamma_{2}\end{array}\right\}$

where in-plane strains are linearly varying through the thickness of the plate (Eq. 2a), whereas the transverse-shear strains are constant along the thickness coordinate, $z$.

\subsection{Experimentally measured membrane strains and bending curvatures}

The strain data can be measured and collected by conventional strain gauges or Fibre Bragg Grating (FBG) strain sensors. The sensors should be mounted at the upper and lower surfaces of the plate, which are represented by + and - signs, respectively. For each iQS4 element, the in situ membrane strains and bending curvatures can be calculated at a particular in-plane location $\boldsymbol{x}_{i}(i=1, \ldots, n)$ by using the surface strain measurements collected from the same $\boldsymbol{x}_{i}$ position as [23]:

$\boldsymbol{E}_{i}=\left\{\begin{array}{l}E_{1} \\ E_{2} \\ E_{3}\end{array}\right\}_{i}=\frac{1}{2}\left\{\begin{array}{l}\varepsilon_{x x}^{+}+\varepsilon_{x x}^{-} \\ \varepsilon_{y y}^{+}+\varepsilon_{y y}^{-} \\ \gamma_{x y}^{+}+\gamma_{x y}^{-}\end{array}\right\}_{i}(i=1, \ldots, n)$

and 
$\boldsymbol{K}_{i}=\left\{\begin{array}{l}\mathrm{K}_{1} \\ \mathrm{~K}_{2} \\ \mathrm{~K}_{3}\end{array}\right\}_{i}=\frac{1}{2 h}\left\{\begin{array}{l}\varepsilon_{x x}^{+}-\varepsilon_{x x}^{-} \\ \varepsilon_{y y}^{+}-\varepsilon_{y y}^{-} \\ \gamma_{x y}^{+}-\gamma_{x y}^{-}\end{array}\right\}_{i}(i=1, \ldots, n)$

where the symbol $n$ denotes the total number positions at which two sets of strain sensors are available within the body of the iQS4 element. The in situ section strains given in Eqs. (3a-b) are experimental counterparts of the analytical section strains given in Eqs. (1a-b), respectively. Note that these discrete section strains, i.e., $\boldsymbol{E}$ and $\boldsymbol{K}$ where the subscript $i$ is naturally dropped to highlight the continuity throughout the element domain, can be fitted into continuous functions through smoothing or curve fitting approaches. The transverse shear strains $\boldsymbol{\Gamma}$ cannot be directly calculated by using the surface strain measurements. Fortunately, these section strains can be easily obtained for isotropic materials through equilibrium equations of Mindlin plate theory by using the derivatives of the $\boldsymbol{E}$ and $\boldsymbol{K}$ section strains as explained in [1]. However, since most of the offshore structures are classified as thinwalled structures, the transverse shear strains are much smaller than the in-plane strains, hence the contribution of $\boldsymbol{\Gamma}$ strains can be safely neglected during the iFEM analysis.

\subsection{Weighted least-squares formulation for iQS4 element}

The difference between the analytical, $\boldsymbol{e}, \boldsymbol{\kappa}$, and $\boldsymbol{\gamma}$, and the experimental, $\boldsymbol{E}, \boldsymbol{K}$, and $\boldsymbol{\Gamma}$, section strains for each iQS4 element can be defined using error functions of $\boldsymbol{\varphi}_{i}\left(\boldsymbol{u}_{e}\right)(i=m, b, s)$, respectively, as:

$$
\begin{aligned}
& \varphi_{m}\left(\boldsymbol{u}_{e}\right)=\boldsymbol{e}\left(\boldsymbol{u}_{e}\right)-\boldsymbol{E} \\
& \boldsymbol{\varphi}_{b}\left(\boldsymbol{u}_{e}\right)=\boldsymbol{\kappa}\left(\boldsymbol{u}_{e}\right)-\boldsymbol{K} \\
& \boldsymbol{\varphi}_{s}\left(\boldsymbol{u}_{e}\right)=\boldsymbol{\gamma}\left(\boldsymbol{u}_{e}\right)-\boldsymbol{\Gamma}
\end{aligned}
$$

The iFEM/iQS4 methodology establishes a weighted-least-squares functional by using the error functions given in Eq. (5) as [31]:

$$
\Phi_{e}\left(\boldsymbol{u}_{e}\right)=\frac{1}{V} \iiint_{V}\left(\omega_{m}\left\|\boldsymbol{\varphi}_{m}\left(\boldsymbol{u}_{e}\right)\right\|^{2}+\omega_{b}\left\|\boldsymbol{\varphi}_{b}\left(\boldsymbol{u}_{e}\right)\right\|^{2}+\omega_{s}\left\|\boldsymbol{\varphi}_{s}\left(\boldsymbol{u}_{e}\right)\right\|^{2}\right) d V
$$

where the symbol $\|\cdot\|^{2}$ denotes the squared $\mathrm{L}^{2}$-norms (Euclidean norms) and $V=A \times 2 h$ represents the volume of the element with $A$ defining the mid-surface of the iQS4 element. In Eq. (5), the squared norms of the $\boldsymbol{\varphi}_{i}\left(\boldsymbol{u}_{e}\right)(i=m, b, s)$ vectors can be calculated as the dot product of vectors $\boldsymbol{\varphi}_{i} \cdot \boldsymbol{\varphi}_{i}$. Moreover, in Eq. (5), the symbols $\omega_{m}, \omega_{b}$ and $\omega_{s}$ are the weighting constants corresponding to each individual section strain.

If the experimental values of $\boldsymbol{E}, \boldsymbol{K}$, and $\boldsymbol{\Gamma}$ can be determined and available for the iFEM analysis, all weighting constants will be equal to unity, i.e., $\omega_{m}=\omega_{b}=\omega_{s}=1$. However, if any of these in situ section strains is not available for an iQS4 element in the analysis, a small value of $\alpha=10^{-4}$ 
compared to unity can be assigned as the weighting constant and the least-squares terms will be reduced to the following form:

$$
\begin{gathered}
\left\|\boldsymbol{\varphi}_{m}\left(\boldsymbol{u}_{e}\right)\right\|^{2}=\left\|\boldsymbol{e}\left(\boldsymbol{u}_{e}\right)\right\|^{2}=e_{1}^{2}+e_{2}^{2}+e_{3}^{2} \text { with }\left(\omega_{m}=\alpha\right) \\
\left\|\boldsymbol{\varphi}_{b}\left(\boldsymbol{u}_{e}\right)\right\|^{2}=\left\|\boldsymbol{\kappa}\left(\boldsymbol{u}_{e}\right)\right\|^{2}=\kappa_{1}^{2}+\kappa_{2}^{2}+\kappa_{3}^{2} \text { with }\left(\omega_{b}=\alpha\right) \\
\left\|\boldsymbol{\varphi}_{s}\left(\boldsymbol{u}_{e}\right)\right\|^{2}=\left\|\gamma\left(\boldsymbol{u}_{e}\right)\right\|^{2}=\gamma_{1}^{2}+\gamma_{2}^{2} \text { with }\left(\omega_{s}=\alpha\right)
\end{gathered}
$$

The arrangement made in Eqs. (6a-c) will allow to maintain the robust interpolation connectivity of strain data between iQS4 elements having strain sensors and iQS4 elements having no experimental strain data.

The essence of iFEM methodology is to minimise the $\boldsymbol{\Phi}_{e}\left(\boldsymbol{u}_{e}\right)$ functional with respect to the displacement DOF, $\boldsymbol{u}_{e}$, hence a well match between the analytical and experimentally measured section strains can be obtained. This minimization process can be defined as [31]:

$$
\frac{\partial \boldsymbol{\varphi}_{e}}{\partial \boldsymbol{u}_{e}}=\boldsymbol{k}_{e} \boldsymbol{u}_{e}-\boldsymbol{f}_{e}=0 \rightarrow \boldsymbol{k}_{e} \boldsymbol{u}_{e}=\boldsymbol{f}_{e}
$$

where $\boldsymbol{k}_{e}$ is the coefficient matrix formed by the integration of squared $\boldsymbol{B}^{m}, \boldsymbol{B}^{b}, \boldsymbol{B}^{s}$ matrices over the volume of the element, and $\boldsymbol{f}_{e}$ is a vector constructed by integral of the multiplication of $\boldsymbol{B}^{m}, \boldsymbol{B}^{b}, \boldsymbol{B}^{s}$ matrices with the measured strain data, $\boldsymbol{E}, \boldsymbol{K}$ and $\boldsymbol{\Gamma}$, through the volume of the element. Note that these integral forms have to be normalized by the volume of the element to attain units of strain measures. The mathematical expressions of the matrix, $\boldsymbol{k}_{e}$ and vector, $\boldsymbol{f}_{e}$ are explicitly given in [31]. Before performing the assembly process for the discretized structure, it is necessary to use the elemental transformation matrix to transform the local matrix-vector equation for each iQS4 element into global system of equations of the whole structure. After applying the specific boundary conditions to avoid singularities (e.g., rigid body motion mode), finally the displacements and rotations of each node can be calculated for any loading condition.

\subsection{Damage identification}

After following the procedure described in Sections 2.2-2.4, the displacements and rotations corresponding to six DOF of each node can be obtained and this data can be subsequently used to calculate full-field section strain measures as given in Eqs. (1-2). Moreover, constitutive relationship between stress and strain will yield the full-field stress distribution. In this study, von Mises strain $\varepsilon_{v m}$ is considered as an equivalent maximum strain to predict damage. This equivalent strain can be calculated under plane stress condition as:

$$
\varepsilon_{v m}=\frac{1}{\sqrt{2}} \sqrt{\left(\varepsilon_{x x}-\varepsilon_{y y}\right)^{2}+\left(\varepsilon_{x x}-\varepsilon_{z z}\right)^{2}+\left(\varepsilon_{y y}-\varepsilon_{z z}\right)^{2}+6 \gamma_{x y}^{2}}
$$

with 
$\varepsilon_{z z}=\frac{v}{v-1}\left(\varepsilon_{x x}+\varepsilon_{y y}\right)$

where $v$ is Poisson's ratio of the material.

In order to clearly show and distinguish the locations of the damage, an additional damage parameter $D$ is introduced and defined as:

$$
D=\left|\frac{\varepsilon_{v m, \text { current }}-\varepsilon_{v m, \text { undamaged }}}{\varepsilon_{v m, \text { undamaged }}}\right|
$$

where $\varepsilon_{v m, \text { current }}$ represents the current measured von Mises strain value and $\varepsilon_{v m, \text { undamaged }}$ denotes the von Mises strain value measured earlier for an undamaged (intact) structure. Note that although the definition of the damage parameter has a similar form to the anomaly index given in Colombo et al. [46], it has a different meaning. If there is no damage inside the structure, it is expected that the value of the damage parameter $D$ is zero everywhere inside the structure as long as there is no rapid change in loading acting on the structure. If there is a rapid change in loading acting on the structure, then there is a possibility that the damage parameter $D$ values can be non-zero. To overcome this problem, once non-zero damage parameter $D$ situation occurs, von Mises strain distribution should also be checked since higher von Mises strain values localise and occur around the damaged areas. Therefore, in this study, both the damage parameter $D$ and von Mises strain distributions are utilised for dent damage identification in stiffened cylindrical structures.

\section{Numerical results}

To demonstrate the capability of iFEM for damage prediction, a longitudinally and transversely stiffened thin-walled cylindrical structure, which was previously considered by Cerik [39], was analysed after introducing local dent damage. The length of the cylindrical structure is $10 \mathrm{~m}$ and it has a circular cross-section with a radius of $3 \mathrm{~m}$. There are 12 longitudinal stiffeners with a web height of $200 \mathrm{~mm}$ at the inner surface. Besides, there are also three ring stiffeners, which have the same web height with the longitudinal stiffeners in the circumferential direction, to provide additional enhancement to the structure. The cylinder and the stiffeners are made from high tensile strength steel with Young's modulus of $210 \mathrm{GPa}$ and Poisson's ratio of 0.3 . The structure can be considered as a thin-walled cylinder and the thickness of the cylinder including the stiffeners is $25 \mathrm{~mm}$. There is an initial geometrical defect representing the local dent with a width of $1 \mathrm{~m}$ and a depth of $0.1 \mathrm{~m} \mathrm{[39].}$ The material degradation approach [47] is utilized to represent the pre-existing damage in a rather simple way. Therefore, Young's modulus of the material is assumed to be $210 \mathrm{kPa}$ in the damage zone. The cylinder is fully fixed at the far edge and all DOFs at the front edge are constrained except the displacement along the axial direction, $x$. A tensional displacement of $0.005 \mathrm{~m}$ is applied in the axial direction to the front edge boundary as the loading condition. 
Table 1. Case Studies for iFEM analysis

\begin{tabular}{|c|c|}
\hline Cases & Damage Location \\
\hline Case1 & No damage on the cylinder \\
\hline Case2 & Central damage at the joint of the stiffeners \\
\hline Case3 & Central damage crossing two longitudinal stiffeners \\
\hline Case4 & Damage at a quarter length of the cylinder \\
\hline Case5 & Multiple damages at edges and centre \\
\hline
\end{tabular}

As listed in Table 1, five different numerical cases examined in this section including Case1 representing no damage case whereas Case 2 to Case 5 represent different damage cases. The damage locations are depicted in Figure 3.

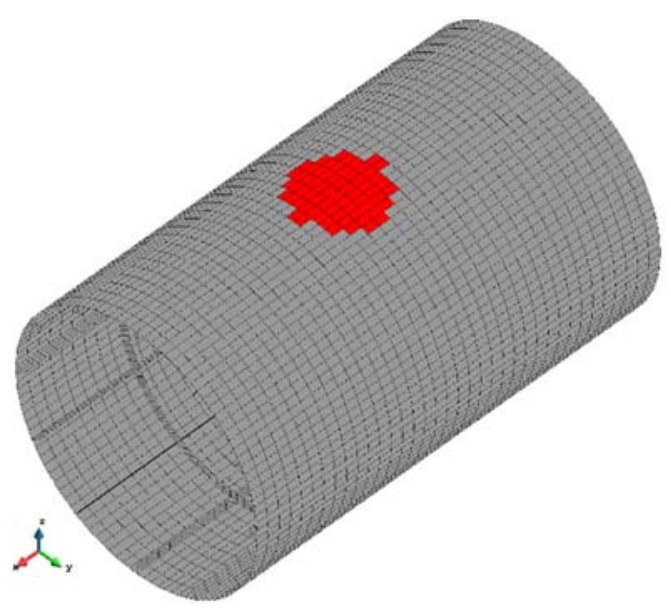

(a)

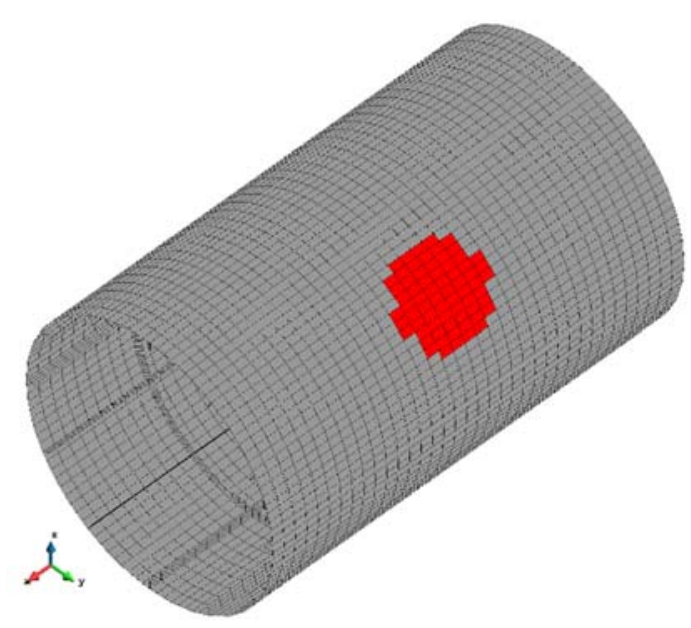

(b)

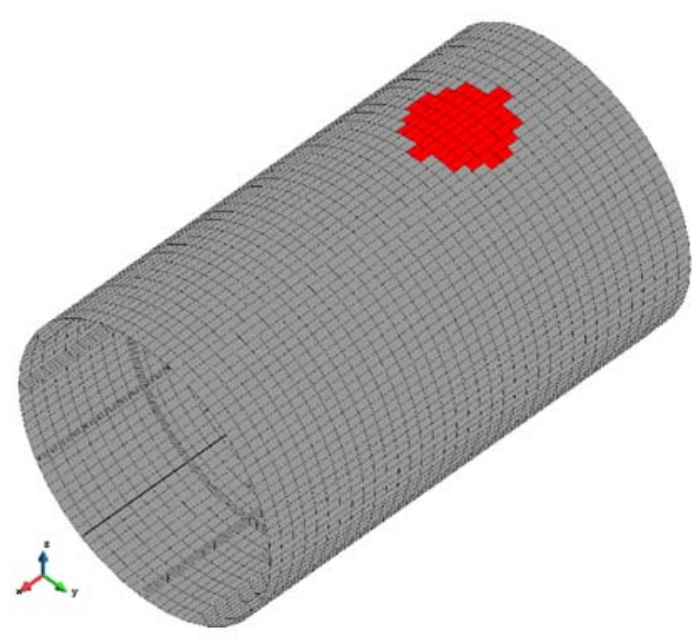

(c) 


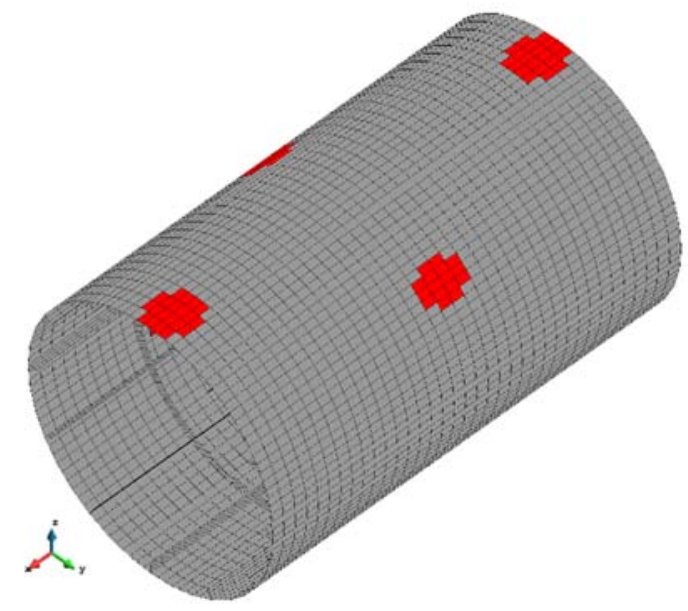

(d)

Figure 3. Damage locations for (a) Case2, (b) Case3, (c) Case4, and (d) Case 5

The main aim of Case 1 is to validate the iFEM methodology for stiffened cylindrical structures, investigate the possibility of reducing sensors under the premise of obtaining reasonably accurate results and obtain reference undamaged solution to calculate the damage parameter, $D$. For the remaining four cases (Case2-5), the damage prediction study is performed for the cylindrical structure using both full sensors and reduced number of sensors. Through this procedure, we will investigate the practicality of iFEM methodology for accurate and reliable detection of dent damage locations even with sparse strain data. Note that in this study, the strain data is generated numerically by performing a high-fidelity FEM analysis by using ANSYS, a commercially available finite element software. A very fine mesh of the cylinder model composed of 6156 elements (Figure 4a) is utilized in the FEM analysis whereas the iFEM analysis is performed by using an in-house MATLAB code with a relatively coarse mesh having only 684 iQS4 elements (Figure 4b).

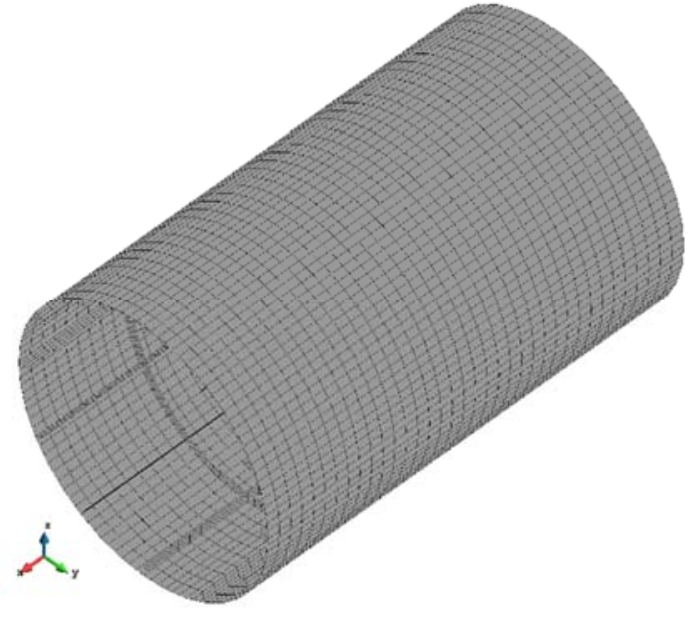

(a)

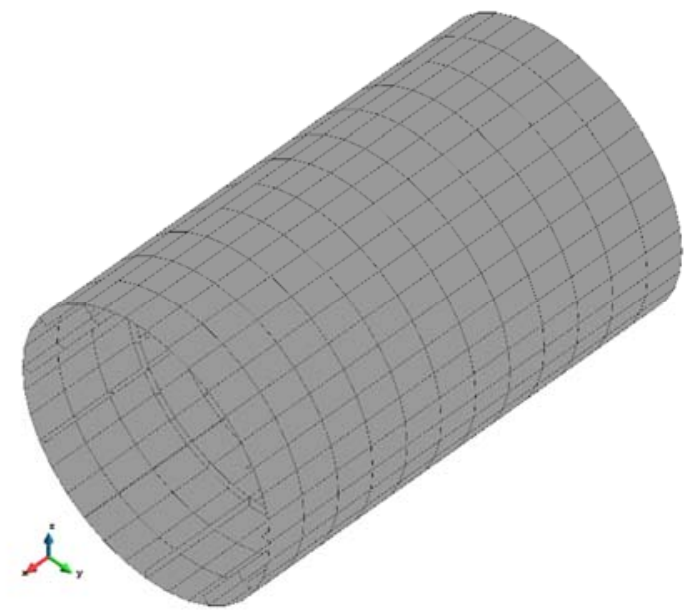

(b)

Figure 4. (a) Dense mesh for FEM model and (b) Coarse mesh for iFEM model 
In this section full-sensor placement means that every iQS4 element has corresponding experimental strain measurements which are provided by FEM analysis. If the full-sensor placement is used, the number of strain sensors becomes $684 \times 2$. It is beneficial to reduce the number of sensors for practical applications. Therefore, iFEM model of stiffened cylindrical structures using less number of sensors, a reduced-sensor model, which contains $192 \times 2$ sensors, is also considered as shown in Figure 5 . The selected iQS4 elements are highlighted in blue.

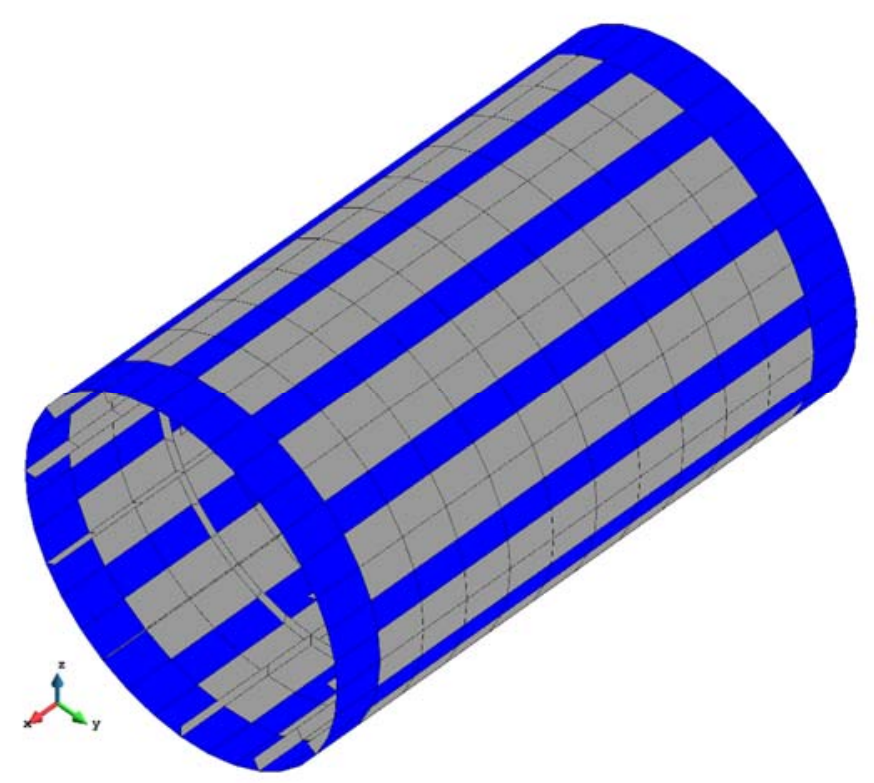

Figure 5. Sensor locations for the reduced sensor model

Twelve longitudinal lines of sensors are used to monitor the whole structure and another two transverse lines of sensors are placed at the boundary of the structure. Note that the number of the sensors can be further reduced, but after balancing the requirement of accuracy and the desire to reduce the number of sensors, the current strategy of sensor installations is preferred. More importantly, thanks to the development of Fibre Bragg Grating (FBG) sensor technology, the proposed number of strain data can be collected without much difficulty. As shown in Figure 5, the sensor locations of reduced-sensor model follow continuous paths over the cylindrical surface. Thus, the strain data can be collected from these locations using several fibre optic cables only. For the current structure and analysis, maximum of 16 FBG sensors can fulfill the requirement of strain data collection.

As mentioned earlier, in this study, strain data for iFEM analysis is provided from a FEM model. Moreover, FEM results are also used to verify the iFEM model for the case without damage. Variations of the total displacements, $\mathrm{U}_{\mathrm{T}}$, along the stiffened cylindrical structure are obtained both by using iFEM full sensor (iFEM) and reduced-sensor (iFEM-r) cases. As shown in Figure 6, both full sensor and reduced sensor cases successfully capture the displacement variation obtained by using FEM. Von Mises strain variation obtained using FEM is also evaluated and presented in Figure 7. These von Mises strain values will be considered as the von Mises strain values for the undamaged case to be used to calculate the damage parameter given in Eq. (10). 


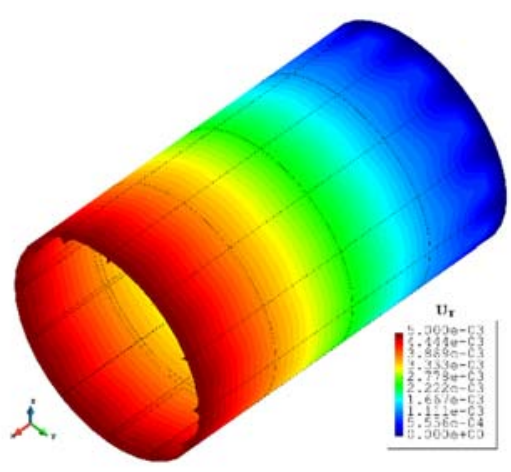

(a) FEM

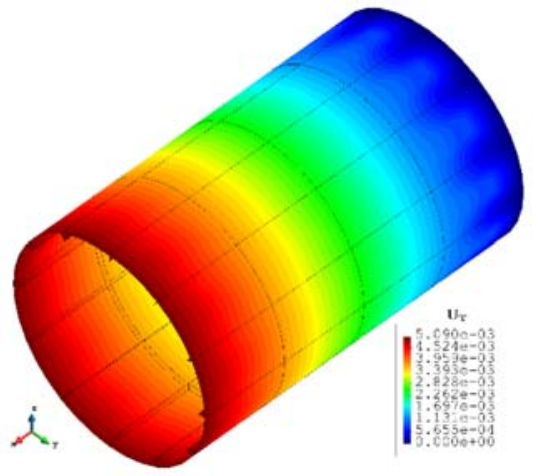

(b) iFEM

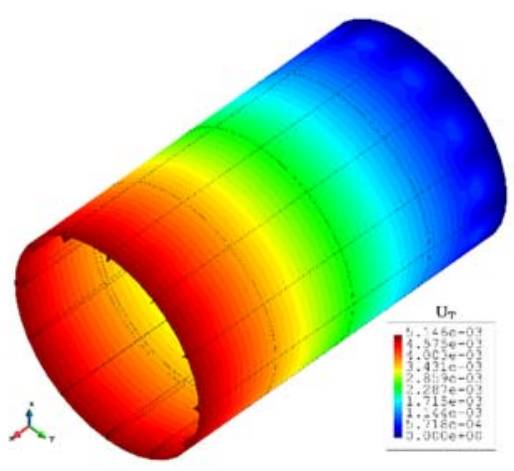

(c) iFEM-r

Figure 6. Contour plot of $U_{\mathrm{T}}$ displacement (unit: $\mathrm{m}$ ) for Case 1

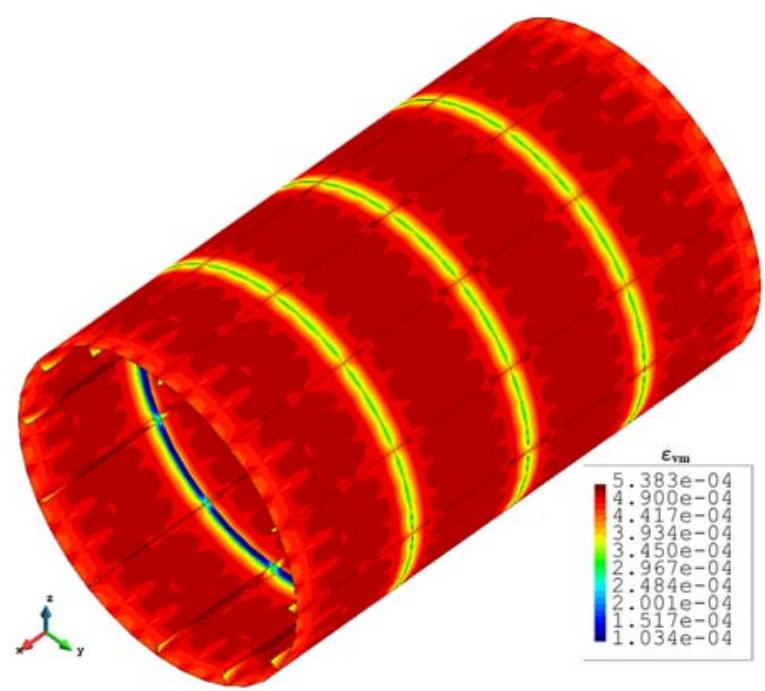

Figure 7. Contour plot of $\varepsilon_{v m}$ strain for FEM of Case 1

In the first damage case, a central damage located at the joint of the stiffeners is considered as shown in Figure 3a. The damage parameter variation for full sensor and reduced sensor cases are given in Figure 8. As can be seen in this figure, the damage parameter takes very large values at the damage location region. Therefore, it is expected that the damage is likely existing in this region. This conclusion is also supported by the von Mises strain distributions demonstrated in Figure 9. Although strain localisation is less visible for reduced sensor case, both full sensor and reduced sensor cases identify the same damage location as the damage parameter.

In the second damage case, a central damage crossing two longitudinal stiffeners is considered as depicted in Figure 3b. The damage parameter values obtained from iFEM analysis for both full sensor and reduced sensor cases are presented in Figure 10. Similar to the previous case, iFEM identified the damage location accurately although reduced sensor case predicted slightly larger damage zone. Moreover, von Mises strain distributions given in Figure 11 obtained from iFEM analysis also clearly demonstrate strain localisations at the damage region. 


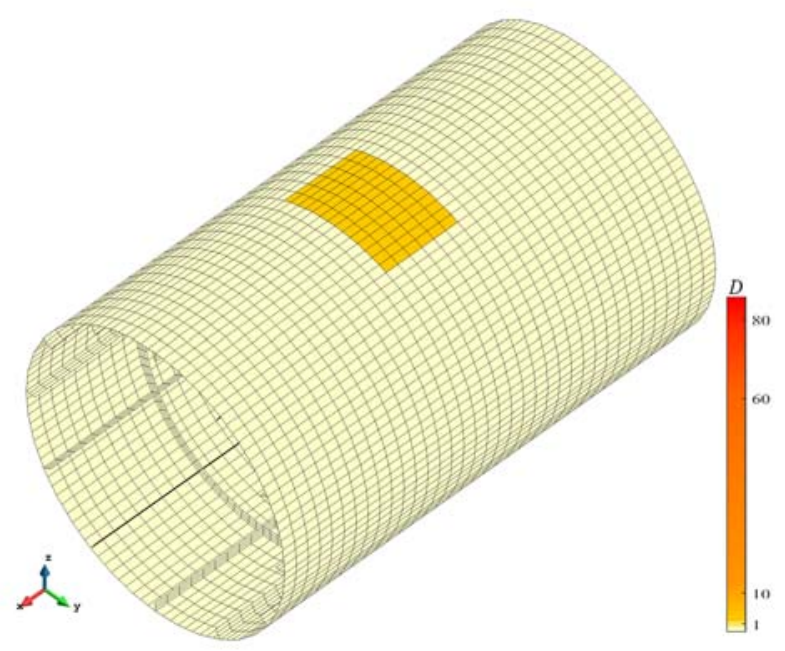

(a) iFEM

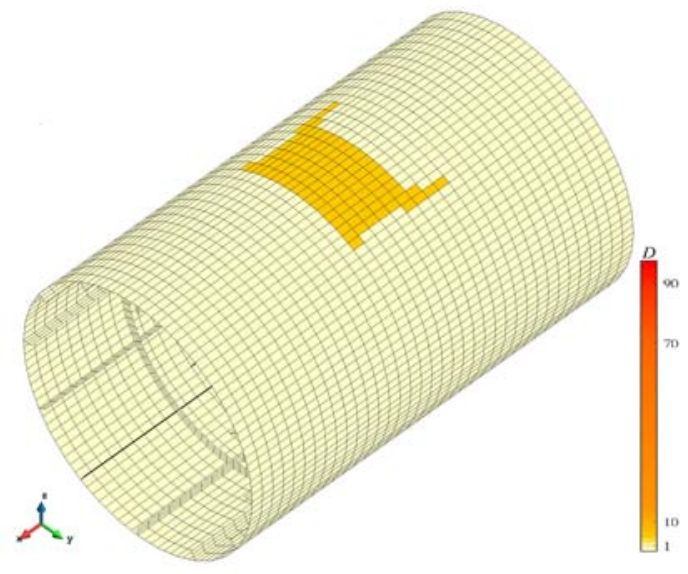

(b) iFEM-r

Figure 8. Damage parameter variation for Case2

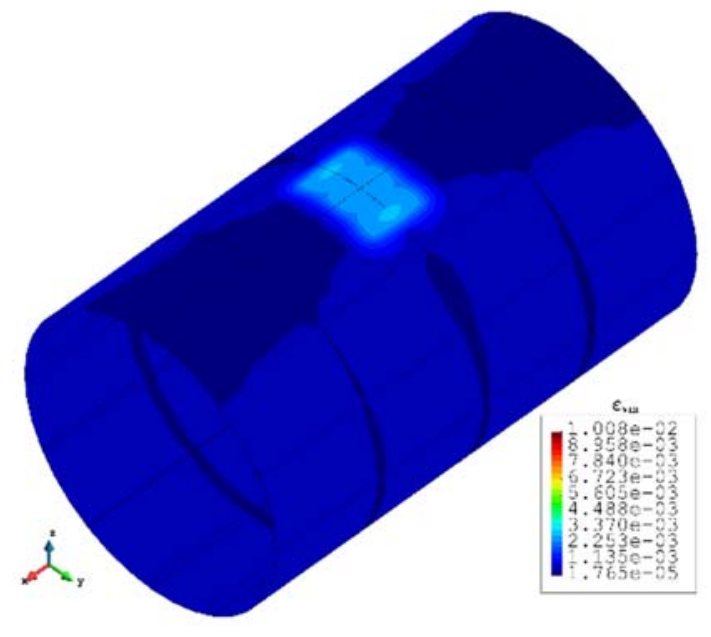

(a) iFEM

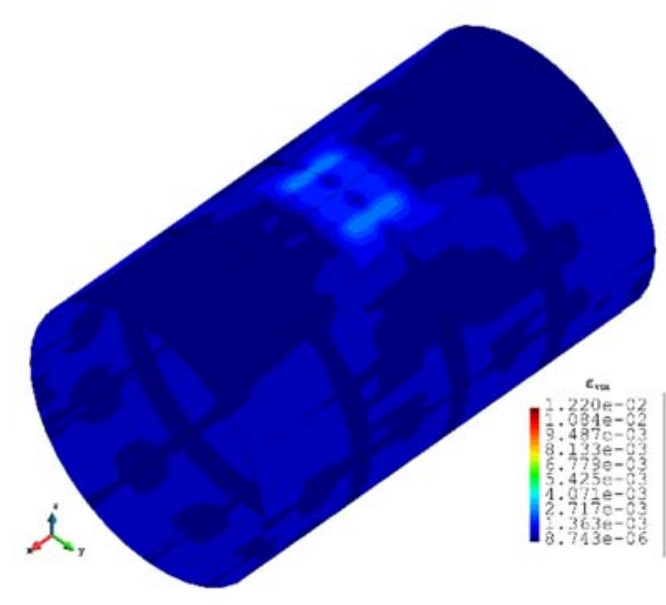

(b) iFEM-r

Figure 9. Von Mises strain variation for Case2 


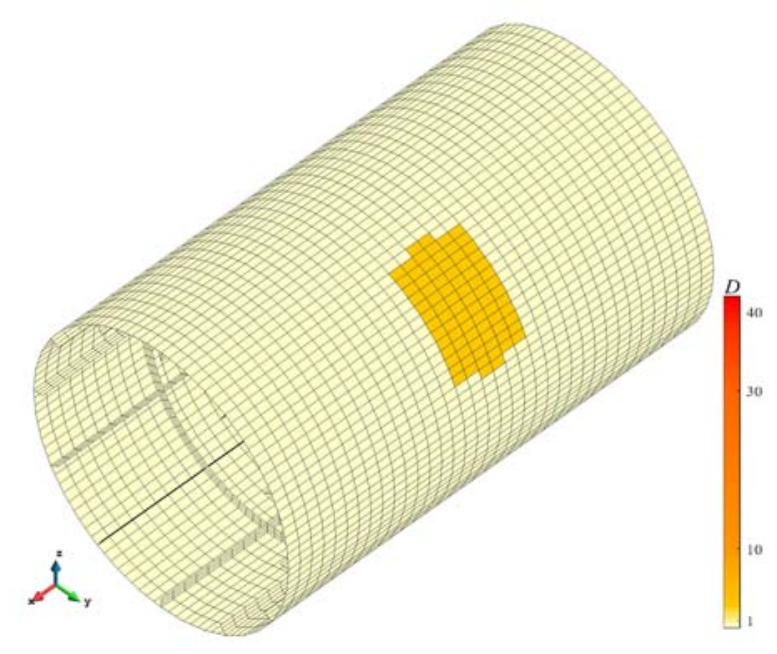

(a) iFEM

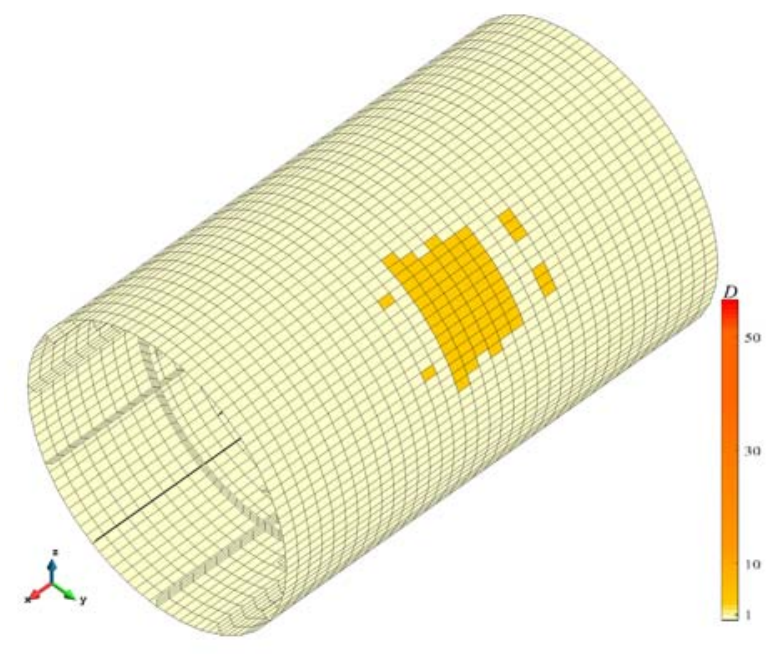

(b) iFEM-r

Figure 10. Damage parameter variation for Case3

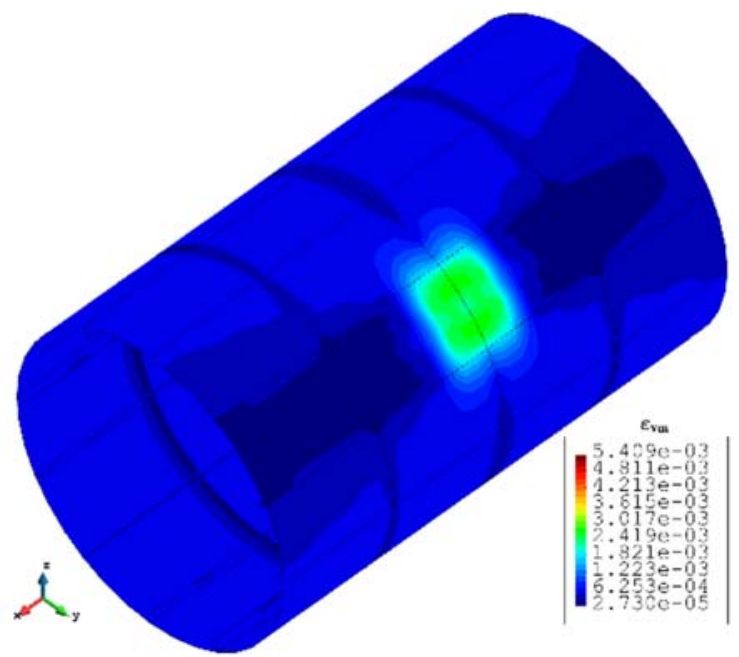

(a) iFEM

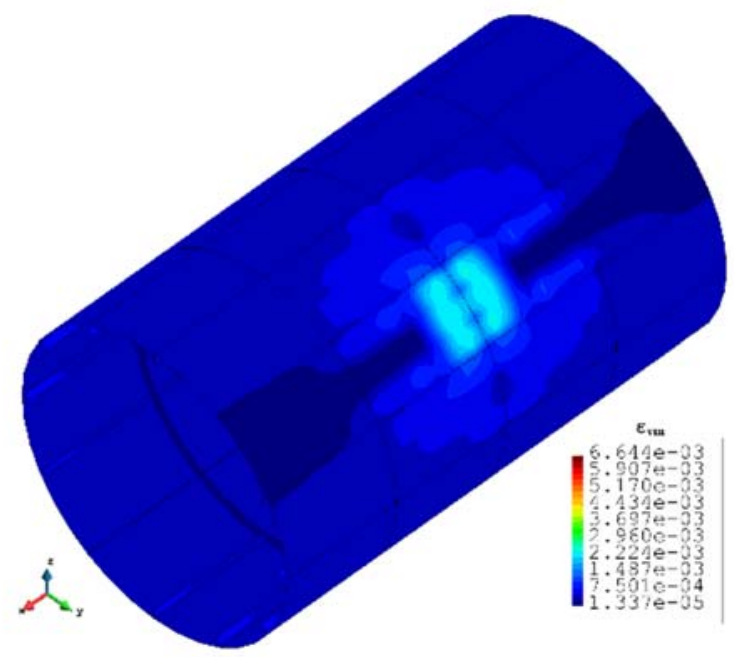

(b) iFEM-r

Figure 11. Von Mises strain variation for Case3

As shown in Fig. 3c, the damage is located closer to the fixed boundary for the third damage case. As can be seen in Figure 12, based on the damage parameter variations obtained from iFEM analysis, the correct damage location can be easily identified. The von Mises strain distributions given in Figure 13 also support this conclusion. As the final damage case, multiple and smaller damages located at different parts of the cylindrical structure are considered as depicted in Fig. 3d. Damage parameter variation for both full sensor and reduced sensor cases are given in Figure 14. iFEM performs also very well for this complex scenario. All damage locations are successfully captured. Von Mises strain distributions also highlight the same damage locations clearly as shown in Figure 15.

As a summary, for all four different damage cases with different size, location and number of damages, iFEM was able to capture the correct damage locations by utilising the newly introduced damage parameter and von Mises strain distribution. 


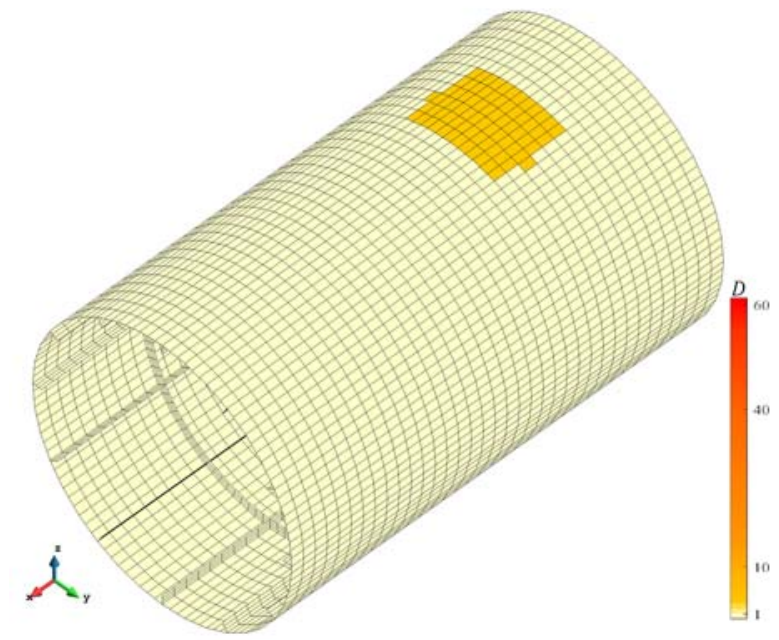

(a) iFEM

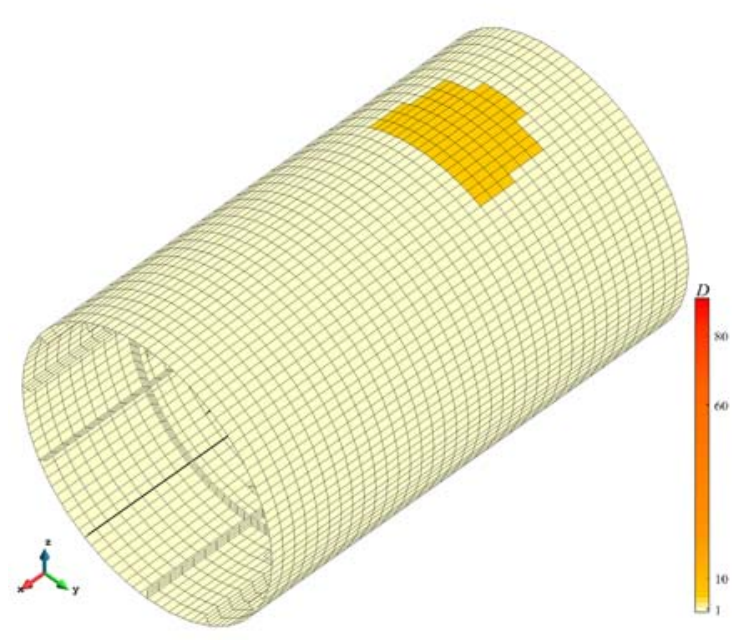

(b) iFEM-r

Figure 12. Damage parameter variation for Case4

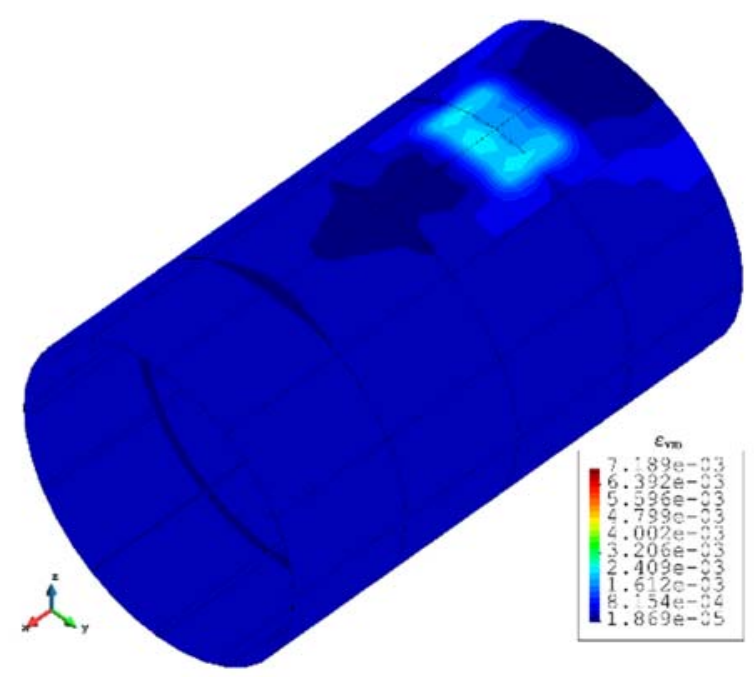

(a) iFEM

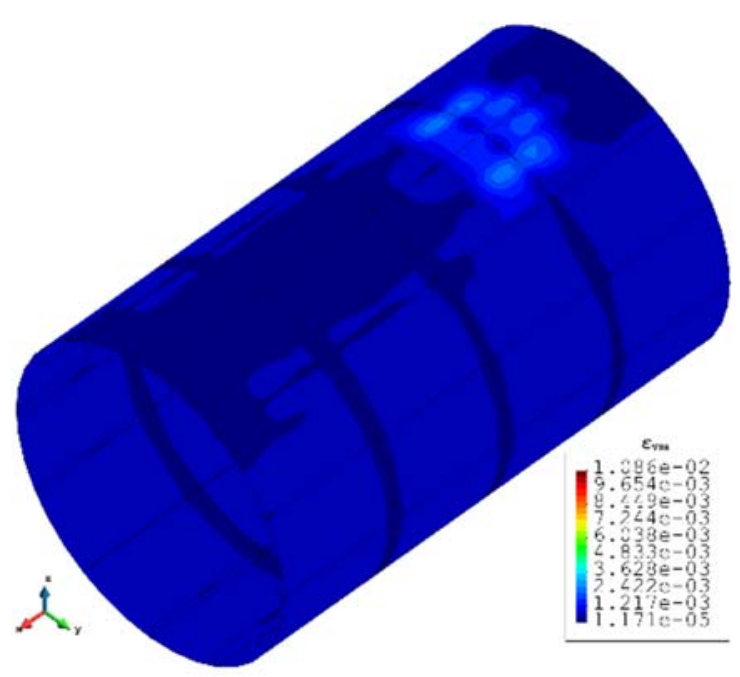

(b) iFEM-r

Figure 13. Von Mises strain variation for Case4 


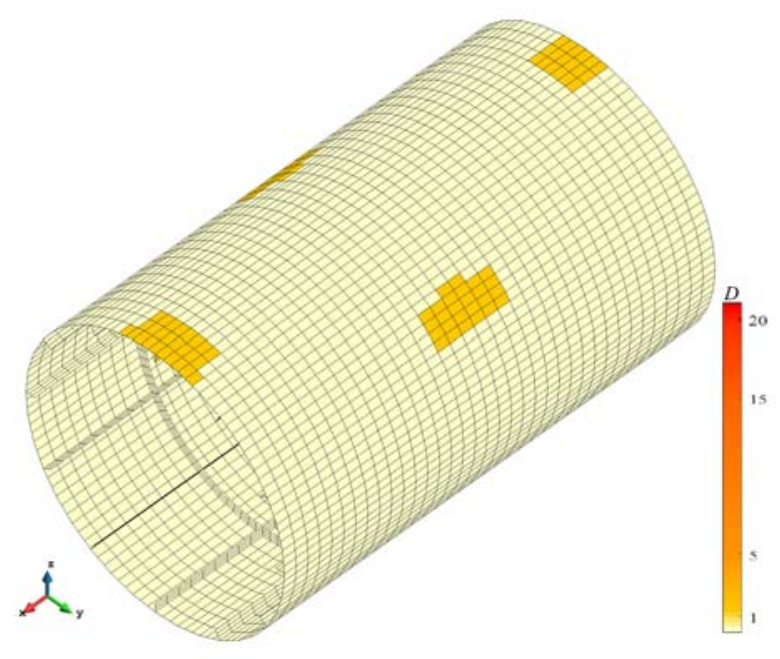

(a) iFEM

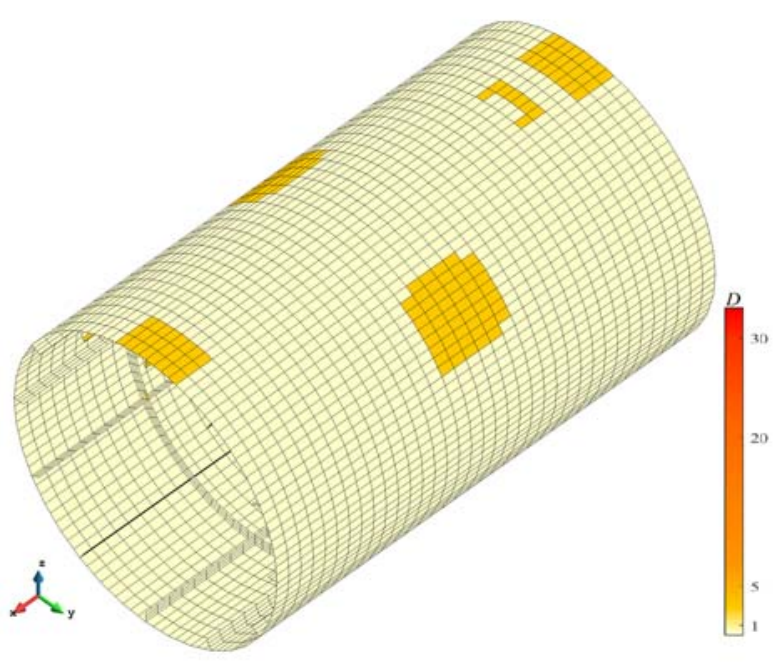

(b) iFEM-r

Figure 14. Damage parameter variation for Case5

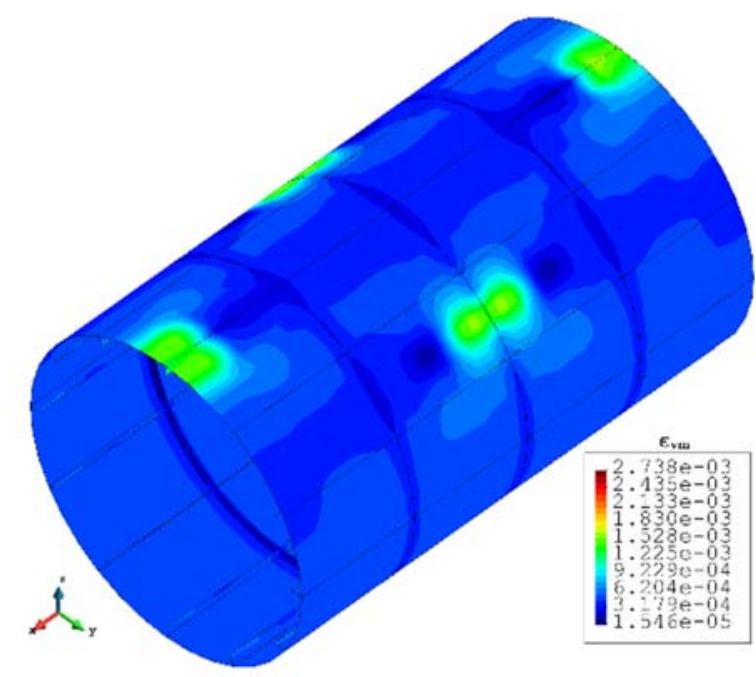

(a) iFEM

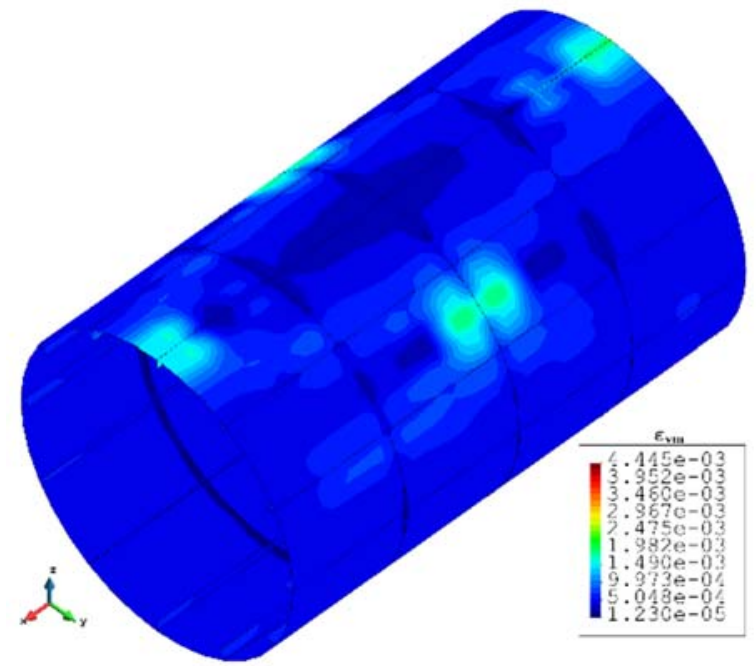

(b) iFEM-r

Figure 15. Von Mises strain variation for Case5

\section{Conclusions}

In this study, inverse Finite Element Method (iFEM) was utilised to monitor and predict structural damage in terms of geometrical defects in thin-walled stiffened cylindrical structures. The stiffened cylindrical structure is modelled with dense mesh for FEM analysis and coarse mesh for iFEM analysis. A robust iFEM shell element-iQS4 is utilized to generate the model for inverse analysis and at the same time FEM results are used both to provide strain data for iFEM analysis and for validation purposes. Five different numerical cases were considered. First of all, based on the displacement results obtained from iFEM analysis of an undamaged structure, it was shown that iFEM 
can easily and accurately perform accurate SHM process for offshore cylindrical structures. In addition, four different cases with damages were investigated to explore the capability of iFEM for predicting the damage locations and shapes. The four cases include damage at the central and quarter ring lines of the structure, small and multiple random damages. Regardless of changing the locations of the damages or varying the sizes of the damage, iFEM can obtain accurate results. Besides, the damage locations can be correctly detected using the damage parameter and von Mises strain plots even with the reduced sensor configuration. Finally, it can be concluded that iFEM is suitable for real-time structural health monitoring and damage prediction in offshore cylindrical structures with high accuracy.

Acknowledgments: Adnan Kefal and Erkan Oterkus greatly acknowledge the financial support provided by TUBITAK under the grant No: 217M207.

Conflicts of Interest: The authors declare no conflict of interest.

\section{References}

1. Kefal, A. Structural Health Monitoring of Marine Structures by Using Inverse Finite Element Method. PhD thesis, University of Strathclyde, Glasgow, UK, 2017.

2. Glisic, B., Inaudi, D. Fibre Optic Methods for Structural Health Monitoring. John Wiley \& Sons: Chichester, UK, 2008.

3. Gherlone, M.; Cerracchio, P.; Mattone, M. Shape sensing methods: Review and experimental comparison on a wing-shaped plate. Prog. Aerosp. Sci. 2018, 99, 14-26.

4. Ko, W.L.; Richards, W.L.; Fleischer, V.T. Applications of the Ko displacement theory to the deformed shape predictions of the doubly-tapered Ikhana wing. NASA/TP-2009-214652, 2009.

5. Arritt, B.; Murphey, T.; Dumm, H.P.; Polland, E.; Klimcak, C. Demonstration of the use of fiber-optics, with integrated fiber-bragg gratings, for shape determination of large deployable strucutres. In: Proceedings of the 48th AIAA/ASME/ASCE/AHS/ASC Structures, Structural Dynamics, and Materials Conference, Honolulu, AIAA 2007-2006, 2007.

6. Blandino, J.R.; Duncan, R.G.; Nuckels, M.C.; Cadogan, D. Three-dimensional shape sensing for inflatable booms. In: Proceedings of the 46th AIAA/ASME/ASCE/AHS/ASC Structures, Structural Dynamics and Materials Conference, Austin, AIAA 2005-1807, 2005.

7. Nishio, M.; Mizutani, T.; Takeda, N. Structural shape reconstruction with consideration of the reliability of distributed strain data from a Brillouin-scattering-based optical fiber sensor. Smart Mater. Struct. 2010, 19, 14-26.

8. Gherlone, M.; Cerracchio, P.; Mattone, M.; Di Sciuva, M.; Tessler, A. Shape sensing of 3D frame structures using and inverse Finite Element Method. Int. J. Solids Struct 2012, 49, 31003112.

9. Bruno, R.; Toomarian, N.; Salama, M., 1994. Shape estimation from incomplete measurements: a neural-net approach. Smart Mater. Struct. 1994, 3, 92-97.

10. Mao, Z.; Todd, M. Comparison of shape reconstruction strategies in a complex flexible structure. In: Proceedings of the SPIE 6932, Sensors and Smart Structures Technologies for Civil, Mechanical, and Aerospace Systems, San Diego, USA, 2008. 
11. Ko, W.L.; Richards, W.L.; Fleischer, V.T. Displacement theories for in-flight deformed shape predictions of aerospace structures. NASA/TP-2007-214612, 2007.

12. Bang, H.J.; Ko, S.W.; Jang, M.S.; Kim, K.I. Shape estimation and health monitoring of wind turbine tower using a FBG sensor array. In: Proceedings of the IEEE Instrumentation and Measurement Technology Conference, Graz, Austria, 2012.

13. Foss, G.; Haugse, E. Using modal test results to develop strain to displacement transformations. In: Proceedings of the 13th International Conference on Modal Analysis, Nashville, USA, 1995.

14. Pisoni, A.C.; Santolini, C.; Hauf, D.E.; Dubowsky, S. Displacements in a vibrating body by strain gauge measurements. In: Proceedings of the 13th International Conference on Modal Analysis, Nashville, USA, 1995.

15. Li, C.J.; Ulsoy, G.A. High-precision measurement of tool-tip displacement using strain gauges in precision flexible line boring. Mech. Syst. Signal Pr. 1999, 13, 531-546.

16. Kang, L.H.; Kim, D.K.; Han, J.H. Estimation of dynamic structural displacements using fiber Bragg grating strain sensors. J. Sound Vib. 2007, 305, 534-542.

17. Rapp, S.; Kang, L.H.; Han, J.H.; Mueller, U.C.; Baier, H. Displacement field estimation for a two-dimensional structure using fiber Bragg grating sensors. Smart Mater. Struct. 2009, 18, 025006.

18. Tessler, A.; Hughes, T.J. An improved treatment of transverse shear in the Mindlin-type fournode quadrilateral element. Comput. Methods Appl. Mech. Eng. 1983, 39, 311-335.

19. Tessler, A.; Spangler, J.L. A variational principle for reconstruction of elastic deformation of shear deformable plates and shells. NASATM-2003-212445, 2003.

20. Tessler, A.; Spangler, J.L. Inverse FEM for full-field reconstruction of elastic deformations in shear deformable plates and shells. In: Proceedings of 2nd European Work-shop on Structural Health Monitoring, Munich, Germany, 2004.

21. Tessler, A., \& Hughes, T. J. (1985). A three-node Mindlin plate element with improved transverse shear. Computer Methods in Applied Mechanics and Engineering, 50(1), 71-101.

22. Quach, C.C.; Vazquez, S.L.; Tessler, A.; Moore, J.P.; Cooper, E.G.; Spangler, J.L. Structural anomaly detection using fiber optic sensors and inverse finite element method. In: Proceedings of AIAA Guidance, Navigation, and Control Conference and Exhibit, San Francisco, California, USA, 2005.

23. Vazquez, S.L.; Tessler, A.; Quach, C.C.; Cooper, E.G.; Parks, J.; Spangler, J.L. Structural health monitoring using high-density fiber optic strain sensor and inverse finite element methods. NASA TM-2005-213761, 2005.

24. Tessler, A.; Spangler, J.L.; Gherlone, M.; Mattone, M.; Di Sciuva, M. Deformed shape and stress reconstruction in plate and shell structures undergoing large displacements: application of inverse finite element method using fiber bragg grating strains. In: Proceedings of 10th World Congress on Computational Mechanics, Sao Paulo, Brazil, 2012.

25. Cerracchio, P.; Gherlone, M.; Mattone, M.; Di Sciuva, M.; Tessler, A. Shape sensing of threedimensional frame structures using the inverse finite element method. In: Proceedings of 5th European Workshop on Structural Health Monitoring, Sorrento, Italy, 2010.

26. Gherlone, M.; Cerracchio, P.; Mattone, M.; Di Sciuva, M.; Tessler, A. Beam shape sensing using inverse finite element method: theory and experimental validation. In: Proceeding of 8th International Workshop on Structural Health Monitoring, Stanford, California, USA, 2011. 
27. Gherlone, M.; Cerracchio, P.; Mattone, M.; Di Sciuva, M.; Tessler, A. An inverse finite element method for beam shape sensing: theoretical framework and experimental validation. Smart Mater. Struct. 2014, 23, 045027.

28. Cerracchio, P.; Gherlone, M.; Di Sciuva, M.; Tessler, A. Shape and stress sensing of multilayered composite and sandwich structures using an inverse finite element method. In: Proceedings of V International Conference on Computational Methods for Coupled Problems in Science and Engineering, Ibiza, Spain, 2013.

29. Cerracchio, P.; Gherlone, M.; Di Sciuva, M.; Tessler, A. A novel approach for displacement and stress monitoring of sandwich structures based on the inverse finite element method. Compos. Struct. 2015, 127, 69-76.

30. Kefal, A.; Hizir, O.; Oterkus, E. A smart system to determine sensor locations for structural health monitoring of ship structures. In: Proceedings of 9th International Workshop on Ship and Marine Hydrodynamics, Glasgow, Scotland, UK, 2015.

31. Kefal, A.; Oterkus, E.; Tessler, A.; Spangler, J.L. A quadrilateral inverse-shell element with drilling degrees of freedom for shape sensing and structural health monitoring. Eng. Sci. Technol. 2016, 19, 1299-1313.

32. American Bureau of Shipping, 1995. Guide for hull condition monitoring systems, 1995.

33. American Bureau of Shipping, 2015. Guide for hull condition monitoring systems, 2015.

34. Det Norske Veritas, Hull Monitoring Systems, DNV Rules for Classification of Ships, Part 6, Chapter 11, 1997.

35. Det Norske Veritas, Hull Monitoring Systems, DNV Rules for Classification of Ships/High Speed, Light Craft and Naval Surface Craft, Part 6, Chapter 11, 2011.

36. Lloyds Register, ShipRight Ship Event Analysis, 2004.

37. Kefal, A.; Oterkus, E. Displacement and stress monitoring of a chemical tanker based on inverse finite element method. Ocean Eng. 2016, 112, 33-46.

38. Kefal, A.; Oterkus, E. Displacement and stress monitoring of a Panamax containership using inverse finite element method. Ocean Eng. 2016, 119, 16-29.

39. Cerik, B.C., Ultimate strength of locally damaged steel stiffened cylinders under axial compression. Thin-Walled Struct 2015, 95, 138-151.

40. Cerik, B. C., Shin, H. K., \& Cho, S. R. (2015). On the resistance of steel ring-stiffened cylinders subjected to low-velocity mass impact. International Journal of Impact Engineering, 84, 108123.

41. Das, P. K., Thavalingam, A., \& Bai, Y. (2003). Buckling and ultimate strength criteria of stiffened shells under combined loading for reliability analysis. Thin-Walled Structures, 41(1), 69-88.

42. Supple, W. J., \& Walker, A. C. (1984). Buckling of offshore structures: a state-of-the-art review. Gulf.

43. Lefebvre, S., \& Collu, M. (2012). Preliminary design of a floating support structure for a 5 MW offshore wind turbine. Ocean Engineering, 40, 15-26.

44. Lai, W.M., Rubin, D.H., Krempl, E. and Rubin, D., 2009. Introduction to continuum mechanics. Butterworth-Heinemann.

45. Irgens, F., 2008. Continuum mechanics. Springer Science \& Business Media. 
46. Colombo, L., Sbarufatti, C. and Giglio, M., 2019. Definition of a load adaptive baseline by inverse finite element method for structural damage identification. Mechanical Systems and Signal Processing, 120, pp.584-607.

47. Taheri-Behrooz, F. and Bakhshan, H., 2018. Characteristic length determination of notched woven composites. Advanced Composite Materials, 27(1), pp.67-83. 